\title{
High Glucose-Induced Vascular Smooth Muscle Cell Proliferation and Migration are Regulated by the miR-34a-Notch1 Pathway
}

\section{Liping Zhang}

Zhejiang University School of Medicine First Affiliated Hospital

\section{Rongrong Pan}

Wenzhou Medical University Affiliated Cixi Hospital: Cixi People's Hospital

Qing Zhang

Zhejiang University School of Medicine First Affiliated Hospital

Linling Gu

Zhejiang University School of Medicine First Affiliated Hospital

\section{Gongde Shi}

Zhejiang University School of Medicine First Affiliated Hospital

Yutao Wu ( $\square$ banshuirentaotao@126.com )

Zhejiang University School of Medicine First Affiliated Hospital

\section{Research Article}

Keywords: high glucose, microRNA-34a, Notch1, vascular smooth muscle cell, proliferation, migration

Posted Date: January 3rd, 2022

DOI: https://doi.org/10.21203/rs.3.rs-1184646/v1

License: () (1) This work is licensed under a Creative Commons Attribution 4.0 International License. Read Full License 
High glucose-induced vascular smooth muscle cell proliferation and migration are regulated by the miR-34a-Notch1 pathway

Liping Zhang ${ }^{1,}$ *, Rongrong Pan ${ }^{2,}$, Qing Zhang, *, Linling Gu${ }^{1}$, Gongde Shi ${ }^{1}$, Yutao $\mathrm{Wu}^{1}$

${ }^{1}$ Department of Cardiology, the First Affiliated Hospital, School of Medicine, Zhejiang University, Hangzhou, Zhejiang 310003, P.R. China

2 Department of Cardiology, CiXi Affiliated Hospital of WenZhou Medical College, NingBo Zhejiang 315300, China

Corresponding to:

Yutao Wu, Department of Cardiology, the First Affiliated Hospital, School of Medicine, Zhejiang University, 79th Qingchun Road, Hangzhou, Zhejiang 310003, P.R. China E-mail: wuyutao@zju.edu.cn;

* Contributed equally

\section{Keywords}

high glucose, microRNA-34a, Notch1, vascular smooth muscle cell, proliferation, migration

\section{Running title}

ZHANG et al: HIGH GLUCOSE-INDUCED VSMC PROLIFERATION AND MIGRATION ARE REGULATED BY MIR-34A-NOTCH1 PATHWAY

\section{Abstract}

High glucose(HG)-induced excessive proliferation and migration of the media vascular smooth muscle cell(VSMC) are the main pathological characteristics in diabetes related vascular injuries. Previous studies have shown that microRNA-34a (miR-34a) is involved in cancer metastasis, proliferation and invasion and plays an essential role in cardiovascular disease. However, little is known about the regulating role miR-34a in HG-induced proliferation and migration of VSMC. Here we demonstrated that miR-34a was downregulated at different timepoints under HG stimulation. Then, HG induced proliferation and migration was found to be impaired 
by miR-34a overexpression using transwell, CCK8 and RT-qPCR assays. Furthermore, the HG-induced depression of "contractile" VSMC-specific markers were reversed by the overexpression of miR-34a. Moreover, we confirmed that miR-34a regulated HG-induced VSMC proliferation and migration through its target gene, Notch1, which has been shown to be associated with cell proliferation and migration in previous studies. Taken together, we propose that the miR34a-Notch1 axis plays an important role in regulating HG-induced VSMC proliferation and migration.

\section{Introduction}

With an exponentially increasing prevalence, diabetes has become a major global health threat. As the main components of the vascular wall, VSMCs are closely related to the occurrence and progression of diabetic atherosclerosis, which is characterized by vascular remodeling, including the phenotypic switching of VSMC from contractile to synthetic phenotype. Vascular remodeling also plays an essential role in other vascular diseases, such as hypertension, vascular injury and arteriosclerosis(1, 2). During vascular remodeling, VSMCs express reduced specific genes, including smooth muscle-actin(SMA), smooth muscle $22 \alpha($ SM22), smooth muscle myosin heavy chain (SMMHC) and h1-calponin, but gain greater capacity in cell proliferation and migration, and produce abundant cytokines and extracellular matrix.

microRNAs(miRNAs) are short, endogenous non-coding RNAs, which regulate gene expression post-transcriptionally(3). miRNAs have been reported to be involved in development(4), differentiation(5), proliferation(6), migration(7), apoptosis(8), metabolism(9), immune response(10) and angiogenesis(11). Recently, increasing studies have revealed that miRNAs play an essential role in cardiovascular disease through regulating VSMC phenotypic transition. For instance, Albinsson et al.(12) reported on the essential role of miRNAs in VSMC in deleting the rate-limiting enzyme in miRNA synthesis. miR-143/145 cluster was reported to be a molecular key in the phenotype switching of VSMC(12). Moreover, miR-21 can regulate VSMC function via targeting tropomyosin 1 in arteriosclerosis obliterans of the lower 
extremities(13). More recently, a study in our department demonstrated that miR-22 was related to VSMC phenotypic modulation and neointima formation by targeting MECP2 and EVI-1(14). These findings suggested the significant roles of miRNAs in vascular disease and provided novel insights into studying the phenotypic transition of VSMC.

miR-34a is a well-studied miRNA in cancer metastasis, proliferation and invasion. miR-34a acts as a suppressor of neuroblastoma tumorigenesis by directly targeting E2F3 mRNA, which is a potent transcriptional inducer of cell-cycle progression and leads to the induction of a caspase-dependent apoptotic pathway(15). Different studies have demonstrated that p53 can bind directly to the precursor gene of miR-34a and activate its transcription during p53-induced apoptosis and G1-arrest(16, 17). Zenz et al.(19) suggested miR-34a was part of the resistance network in chronic lymphocytic leukemia. Recently, our group reported that miR-34a played an important role both in VSMC differentiation from stem cells and in vessel injury-induced neointima formation(18). In another study, down-regulation of miR-34a was associated with the alleviation of mesangial proliferation and glomerular hypertrophy by targeting growth arrest-specific1(GAS1)(19). Notch signaling pathways are important targets of miR-34a. High levels of miR-34a suppress the self-renewal of colon cancer stem cells through the down-regulation of Notch expression(20). These findings have revealed a vital role of miR-34a in regulating cell fate. However, the role of miR-34a in VSMC proliferation and migration induced by high glucose $(\mathrm{HG})$ levels, and the related signaling pathways, remain elusive. In this study, we demonstrated for the first time that miR-34a inhibits HG-induced VSMC proliferation and migration through targeting Notch1.

\section{Materials and Methods}

\section{Cell isolation and culture}

Primary mouse VSMCs were isolated from C57BL/6 mice (purchased from the Shanghai Institutes for Biological Sciences, Shanghai, China ) aorta, and cultured in DMEM (Genom, Hangzhou, China) containing $5.5 \mathrm{mmol} / \mathrm{L}$ glucose and 
supplemented with 10\% fetal bovine serum ( FBS: cat. no. 10099141; Gibco; Thermo Fisher Scientific, Inc., Waltham, MA, USA) as described previously(18). Anesthesia was induced and maintained using 100\% O2 / 4\% isoflurane. All mice were euthanized by deep anesthesia with $100 \% \mathrm{O} 2$ / 5\% isoflurane, followed by dislocated execution. All animal experiments were conducted according to the ARRIVE guidelines. All the animal procedures were approved by the National Institutes of Health Guide for the Care and Use of Laboratory animals(NIH Publications No. 8023, revised 1978). DMEM containing $25 \mathrm{mmol} / \mathrm{L}$ glucose was used as the HG medium in this study, and DMEM containing $5.5 \mathrm{mmol} / \mathrm{L}$ glucose was defined as normal glucose medium(NG). Cells between passages 5 and 10 were sampled for assays. In all assays, cells were serum-starved for 12-24 hours without FBS and then subjected to the NG or HG with $10 \%$ FBS.

\section{VSMC proliferation and migration assays}

To verify the effect of HG on VSMC proliferation and migration, the primary VSMCs were cultured in NG or HG medium for 4, 8 and 12 hours and subjected to further studies. Four groups including $\mathrm{NG}+$ mimic normal control (NC) , NG+miR-34a mimic, $\mathrm{HG}+$ mimic $\mathrm{NC}$ and $\mathrm{HG}+$ miR-34a mimic were set to detect the role of miR-34a in HG-induced VSMC proliferation and migration. Cells were transfected with mimic NC or miR-34a mimic respectively and then cultured in NG or HG medium for 24 hours. VSMC proliferation was evaluated using a Cell Counting Kit-8 (cat. no. C0037; Beyotime, China ) according to the manufacturer's instructions. VSMC migration was evaluated using transwell migration assays(18). In short, the VSMC suspensions were prepared with serum-free medium at the density of $5 \times 10^{5}$ cells $/ \mathrm{ml}$ and the membranes of the Costar Transwell-24 well plates $(8.0 \mu \mathrm{m}$ pore size, cat.no.3422, lifesciences, USA) were coated with Matrigel (cat. no. 356234 ; BD Biosciences, San Jose, CA, USA). $100 \mu \mathrm{l}$ of cell suspension was added to the upper chamber and $600 \mu \mathrm{l}$ of medium containing 20\% FBS was added to the lower chamber. The VSMCs were incubated for 24 hours at $37^{\circ} \mathrm{C}$. The cells were stained with $0.1 \%$ 
crystal violet for 20 min and the upper unmigrated cells were wiped off with a cotton swab before the stained cells were counted under microscope (400x).

\section{miRNAs and siRNA transfection}

Either siRNAs (siRNA-Notch1 and siRNA NC, Ribobio, China, $50 \mathrm{nM}$ ) or miRNAs (50 nM, Baiao, China, miR-34a mimic: forward sequences: 5 ' -UGGCAGUGUCUUAGCUGGUUGU-3', reverse sequences:5'-ACAACCAGCUAAGACACUGCCA-3'; miR-34a inhibitor mimic (miR-34a Int mimic) : 5'-ACAACCAGCUAAGACACUGCCA-3') were transfected into VSMCs using Lipofectamine 3000 (cat. no. L3000-015; Thermo Fisher Scientific, Inc.) using the methodology described previously (5, 21, 22). Briefly, cells were grown in 6-well plates and the growth media replaced with $1.0 \mathrm{ml}$ empty medium without antibiotics or FBS and maintain for 30 min after reaching 60-70\% confluency. The cells were transfected with siRNAs (mixture of A and B; A: $50 \mu 1 \mathrm{DMEM} /$ well+ $1 \mu \mathrm{l}$ siRNA/well; B: $50 \mu \mathrm{l}$ DMEM /well+3.75 $\mu$ l Lipofectamine 3000/well) or miRNAs (mixture of A and B; A: $50 \mu \mathrm{l}$ DMEM /well+ $1 \mu \mathrm{lmiRNA} /$ well; B: $50 \mu 1$ DMEM /well+3.75 $\mu$ l Lipofectamine 3000/well) for 24 hours. The cells were then cultured in NG or HG with 10\% FBS for an additional 24 or 48 hours.

\section{Real time quantitative PCR (RT-qPCR)}

Total mRNA isolation and real-time quantitative PCR (RT-qPCR) were performed as we described previously(23-25). Total mRNAs containing miRNAs were isolated from cells using Trizol (cat. no. 12183555; Invitrogen; Thermo Fisher Scientific, Inc. USA) according to the manufacturer's instructions. Total RNAs and miRNAs specific cDNA synthesis was performed using a PrimeScript RT Master Mix (Perfect Real Time) Kit (cat. no. RR047A; Takara Biomedical Technology Co., Ltd., Beijing, China) and miDETECT A Track ${ }^{\mathrm{TM}}$ miRNA qRT-PCR Starter Kit (cat. no. C10712-1; Ribobio, Guangzhou, China). Real-time PCR for mRNA was performed using Takara premix Ex Taq II (cat. no. DRR820A; Takara Biomedical Technology Co., Ltd, Beijing, China) and was run on ABI Prism 7500 system (Applied Biosystems, USA) in a total volume of $10 \mu$ containing 5 ul Takara premix Ex Taq II 
and 5 ng cDNA template. Real - time PCR for miRNA was performed with miDETECT A Track ${ }^{\mathrm{TM}}$ miRNA qRT-PCR Starter Kit (cat. no. C10712-1; Ribobio) and was run on an ABI Prism 7500 system (Applied Biosystems, USA) in a total volume of $20 \mu \mathrm{l}$ containing 10ul SYBR Green Mix and 10ng cDNA template. The PCR thermal cycling parameters for mRNA were $2 \mathrm{~min}$ at $50 \mathrm{C}, 30 \mathrm{sec}$ at $95 \mathrm{C}, 40$ cycles of $95 \mathrm{C}$ for $5 \mathrm{sec}$ and $60 \mathrm{C}$ for $34 \mathrm{sec}$ and for miRNA were $10 \mathrm{~min}$ at $95 \mathrm{C}$ and 40 cycles of $95 \mathrm{C}$ for $2 \mathrm{sec}, 60 \mathrm{C}$ for $20 \mathrm{sec}$ and $70 \mathrm{C}$ for $10 \mathrm{sec}$. Expression of mRNA/miRNA was normalized to the expression of mouse GAPDH (mGAPDH)/U6 and quantified using the $2^{-\Delta \Delta \mathrm{Cq}}$ method. Primers used in the experiments are listed in the Table I.

\section{Western blot analysis}

Protein extraction and immunoblotting were performed similar to our previous reports $(24,26)$. Briefly, total proteins were extracted from VSMCs using RIPA lysis buffer (cat. no. P0013B; Beyotime, China) and quantified using a BCA Protein Assay Kit (cat. no. P0012S ; Beyotime, China). An equal volume of protein mixed with $5 \mathrm{X}$ SDS loading buffer (cat. no. P0015; Beyotime, China) was boiled for $10 \mathrm{~min}$ at 100 C . 10-30 ug of the protein were separated on 10\% SDS-PAGE gel (cat. no. P0012A; Beyotime, China) and transferred from the gel to the membrane. The membrane was blocked overnight in 5\% skim milk followed by incubation with primary antibodies overnight at $4^{\circ} \mathrm{C}$. The primary antibodies against Notch1 (cat. no. A-8:sc-376403; Santa Cruz Biotechnology, lnc., Dallas, TX, USA; monoclonal antibody from mouse), PCAN (cat. no. 15014-1-AP; D3-3, No.666 Gaoxin Avenue Wuhan East Lake Hi-tech Development Zone Wuhan, Hubei, P.R.C ) and GAPDH (cat. no.14C10; Cell Signaling Technology, lnc.) were diluted 1:1000, 1:500 and 1:1000, respectively. The membrane was washed thrice with $1 \mathrm{X}$ PBST, for 10 min per wash, and incubated with the secondary antibody from Santa Cruz Biotech for 1 hour at room temperature. The membrane was washed again 3 times with 1X PBST. The ECL substrate was added and the target proteins observed with the Compact X4 in the dark room.

\section{Statistical analysis}


All data were analyzed using a two-tailed student's t-test or one-way ANOVA followed by Tukey's HSD multiple comparison post-hoc test.

\section{Results}

miR-34a expression is down-regulated during HG-induced VSMC proliferation and migration

VSMCs cultured in HG media showed a greater growth density than those grown in NG medium after 12 and 24 hours (Figure 1A). Transwell migration assays revealed that HG stimulation promoted VSMC migration when compared against NG (Figure $1 \mathrm{~B}$ and $\mathrm{C}$ ). Furthermore, transcript profiles of miR-34a were downregulated under HG stimulation (Figure 1D), suggesting a role of miR-34a in HG-induced VSMC proliferation and migration.

\section{HG-induced VSMC proliferation and migration may be impaired by miR-34a}

We performed a series of experiments in order to reveal the further role of miR-34a in HG-induced VSMC proliferation and migration. As transwell assay showed, HG-induced migration may be inhibited by miR-34a overexpression (Figure 2A). CCK8 assays also demonstrated that miR-34a overexpression could abolish the proliferation induced by $\mathrm{HG}$ stimulation (Figure 2B). The transcript levels of osteopontin (OPN) and PCAN were determined by RT-qPCR. OPN has been reported to be synthesized by smooth muscle cells in primary and restenotic human coronary atherosclerotic plaques(27) and be associated with cell proliferation(28, 29) and migration(30, 31). PCNA is also expressed in VSMC which has more capacity of proliferation and migration and has been thought to be responsible for the proliferation and migration in many kinds of cells $(32,33)$. As expected, HG induced the upregulation of both OPN and PCAN could be abrated by miR-34a mimic while there was no significance at OPN and PCAN mRNA level between mimic NC group and miR-34a mimic group under NG condition (Figure 2C and D). These results support the hypothesis that miR-34a plays an essential role in regulating VSMC proliferation and migration under HG condition.

HG-induced downregulation of VSMC specific markers is modulated by $\operatorname{miR}-34 a$ 
During the phenotypic switching of VSMC, the increasing capacities for proliferation and migration result in the downregulation of VSMC specific genes, such as SMA, SM22 and SMMHC. Thus, expression of these marker genes was determined by RT-qPCR. HG stimulation inhibited SM22 mRNA expression, while miR-34a overexpression almost abolished this effect (Figure 3A). In contrast, miR-34a Int mimic enhanced the inhibitory effect of HG stimulation (Figure 3B). Meanwhile, the HG-induced downregulation of SMA and SMMHC was affected by miR-34a Int mimic (Figure 3C and D). These data indicated that HG-induced downregulation of VSMC specific markers was modulated by miR-34a.

miR-34a regulates $H G$-induced VSMC proliferation and migration through targeting Notch1

According to TargetScan (http://www.targetscan.org, Figure 4A) and our previous study(18), we examined whether or not miR-34a modulates Notch1 during HG-induced VSMC proliferation and migration. Consistent with our previous study, Notch1 gene and protein levels were significantly downregulated by the overexpression of miR-34a (Figure 4B and C). Conversely, Notch1 levels were significantly upregulated by the inhibition of miR-34a (Figure 4F). Meanwhile, the transfection of siRNA- Notch1 into VSMCs significantly downregulated Notch1 mRNA levels (Figure 4E) and protein levels (Figure 4D). To study the interactions between Notch1 and miR-34a in HG-induced VSMC proliferation and migration further, miR-34a Int mimic and siRNA- Notch1 were co-transfected into VSMCs cultured in HG. Cell counting assays (Figure 4G) and western blotting (Figure 4H) showed that miR-34a Int mimc induced VSMC proliferation and PCAN mRNA overexpression were suppressed by inhibition of Notch1 in these cells. Since we have demonstrated that miR-34a inhibited PCAN expression, VSMC proliferation and Notch1 levels, whereas miR-34a Int mimic reduced these effects, the reduced proliferation in these co-transfected VSMCs was likely due to the downregulated Notch1 levels by its siRNA. Therefore, our results implied that miR-34a modulated HG-induced VSMC proliferation and migration through its target gene Notch1.

\section{Discussion}


Excessive proliferation and migration of VSMCs in the tunica media is one of the main pathological characteristics in diabetes-induced vascular injuries, which leads to the formation of new intima, vascular stenosis and eventually causes disability and death in large numbers of patients. Despite great efforts in research into HG-induced VSMC proliferation and migration in the past decades, a comprehensive molecular mechanism in this pathological process remains elusive. In the present study, we report for the first time that HG-induced VSMC proliferation and migration are regulated by miR-34a. The overexpression of miR-34a impaired VSMC proliferation and migration, and alleviated the HG-induced inhibition of gene expression of VSMC specific markers. Moreover, Notch1 was confirmed to be the target gene of miR-34a in regulating HG-induced VSMC proliferation and migration.

The phenotypic transition of VSMC plays an important role in the physiological and pathological process of vascular complications in type 2 diabetes. However, the precise mechanisms have remained largely unclear. Consistent with several other studies $(34,35)$, HG promoted VSMC proliferation and migration in the present study. As expected, HG induced the expression of OPN and PCAN, which are related to VSMC proliferation and migration(28). Moreover, HG inhibited the expression of SMA, SM22 and SMMHC, which are key markers of VSMC function and are often downregulated in several vascular diseases $(36,37)$. These changes in gene expression may contribute to the impaired normal function and enhanced proliferation and migration in VSMC under HG stimulation. It was also reported that HG can cause oxidative stress and impair apoptosis in VSMC (38-40) and antioxidants can inhibit HG-induced VSMC proliferation and migration(35). However, the apoptosis level of VSMCs induced by HG was not determined in this study.

Decreased miR-34a expression was also observed under HG stimulation. miR-34a is a member of the highly conserved miR-34 family, which plays a major role in regulating cell-cycle progression, apoptosis, and DNA repair in cancer(41-43), neurophysiology and neuropathology studies(44). Recently, miR-34a has been reported to play an essential role in cardiovascular physiological processes and diseases. Shi et al.(39) has reported that miR-34a can promote apoptosis in 
cardiomyocytes and alleviate the injury caused by hypoxia. The research on miR-34a mediated p53 pathways has also deepened our understanding of miR-34a in determining cell fate. p53 can directly bind to the miR-34a precursor gene and modulate its transcription(16, 17, 45). In this study, overexpression of miR-34a largely eliminated the suppressing effects of $\mathrm{HG}$ stimulation on SM22 mRNA expression level while inhibition of miR-34a could enhance the suppressing effects of HG stimulation on SM22 mRNA expression. Meanwhile, inhibition of miR-34a could strengthen inhibitory effect of HG stimulation on SMA mRNA expression though it reduced the inhibitory effect of $\mathrm{HG}$ stimulation on SMMHC mRNA expression. Moreover, overexpression of miR-34a largely eliminated the promoting effects of $\mathrm{HG}$ stimulation on VSMC proliferation and migration ability as transwell and CCK8 assay showed and related genes (OPN and PCAN). Interestingly, miR-34a had no effect on VSMC proliferation and migration under NG condition in our study, suggesting that miR-34a could regulate VSMC proliferation and migration under HG condition rather than under NG condition. This prompts that miR-34a may be an effective and safe therapeutic target for patients suffering diabetes-related vascular diseases. Additionally, the absence of data on VSMC marker genes mRNA expression in the $\mathrm{NG}+$ miR-34a mimic group and protein level of OPN and PCAN is a limitation of our study.

A better understanding of the miRNA targets can help to reveal their complex mechanisms. Notch signaling pathways which were activated in HBZY-1 cells(46)and podocytes(47) exposed to high glucose play prominent roles in VSMC proliferation, migration and apoptosis(48). The targeting of Notch1 by miR-34a has been well studied in glioma cells(49), colon cancer stem cells(20) and breast cancer cells(50). miR-34a can downregulate Notch1 protein levels by binding to mRNA 3'-UTRs(49). Yan Tang et al. has reported that miR-34a inhibits pancreatic cancer progression through regulating epithelial-mesenchymal transition (EMT) by targeting Snaill and Notch1(51), while they do not check the relationship between Snaill and Notch1 under the EMT process. In the previous study from our team(18), Notch1 
repression has been reported to be required for miR-34a mediated VSMC proliferation and migration induced by PDGF or serum. Thus, we guess Notch1 may also act as the functional target of miR-34a under HG stimulation. For the first time, in the present study, we reported that miR-34a overexpression reduced Notch1 mRNA and protein levels in VSMC under HG stimulation while an opposite trend was observed under miR-34a inhibition. Since the data of variation of Notch1 in HG was not completed, it was hard to exclude the effect of HG on the expression level of Notch1. From the existing experimental data and previous studies(46, 47), it is speculated that the effect of miR-34a Int mimic and HG on the expression level of Notch1 should be consistent from the trend. Moreover, In the 'rescue' experiments, the transfection of siRNA- Notch1 into VSMC weaken the miR-34a Int mimic induced PCAN mRNA overexpression and VSMC proliferation. These results indicated that siRNA inhibition of Notch1 resulted in reduced VSMC proliferation. Combining this with the fact that overexpression of miR-34a downregulated Notch1 expression, the targeting of Notch1 by miR-34a in HG-induced VSMC proliferation can be proven. In our present study, we have firstly demonstrated VSMC proliferation and migration under HG condition may be regulated by the miR-34a-Notch1 pathway, which would provide novel insights into diabetes mellitus(DM)-induced vascular complications. However, it is also well acknowledged that miR-34a targets multiple signaling pathways in addition to the Notch1 pathway(20). Hence, the complex mechanism in miR-34a modulated VSMC proliferation and migration requires further study. It is also a limitation that only primary VSMC was used in our study rather than two or more cell lines.

In conclusion, our results provide novel insights into DM-induced vascular complications. We have shown that miR-34a expression is reduced in VSMCs under HG stimulation accompanying increased cell proliferation and migration. Overexpression of miR-34a inhibited VSMC proliferation and migration induced by HG stimulation and alleviated the inhibition on the expression of VSMC specific markers under $\mathrm{HG}$ condition. Moreover, of miR-34a targets and downregulates 
expression of Notch1. Taken together, we conclude that miR-34a regulated HGinduced VSMC proliferation and migration through the miR-34a-Notch1 pathway. Thus, there is potential to develop novel therapeutic strategies in treating DM-induced vascular complications through modulating miR-34a activities by targeting Notch1.

\section{Acknowledgements}

Not applicable

\section{Funding}

This work was supported by grants from the Natural Science Foundation of Zhejiang Province (LY21H020005 and LY16H020009), the Zhejiang Medical Science and Technology Project (2019KY376, 2018KY071), Ningbo Science and technology projects (202002N3173).

\section{Availability of data and materials}

The datasets used and/or analyzed during the current study are available from the corresponding authors on reasonable request.

\section{Authors' contributions}

YW designed the research; LZ, RP, QZ, LG, GS performed the research; YW analyzed the data and wrote the manuscript.

\section{Ethics approval and consent to participate}

Not applicable.

\section{Patient consent for publication}

Not applicable.

\section{Competing interests}

The authors declare that they have no competing interests and all authors should confirm its accuracy

\section{References}

1. Owens GK, Kumar MS and Wamhoff BR: Molecular regulation of vascular smooth muscle cell differentiation in development and disease. Physiol Rev 84: 767-801, 2004. 
2. Rensen SS, Doevendans PA and van Eys GJ: Regulation and characteristics of vascular smooth muscle cell phenotypic diversity. Neth Heart J 15: 100-108, 2007.

3. Bartel DP: MicroRNAs: genomics, biogenesis, mechanism, and function. Ce11 116: 281-297, 2004.

4. Bhaskaran M and Mohan M: MicroRNAs: history, biogenesis, and their evolving role in animal development and disease. Vet Pathol 51: 759-774, 2014.

5. Shi Y and Jin Y: MicroRNA in cell differentiation and development. Sci China C Life Sci 52: 205-211, 2009.

6. Lee J, Heo J and Kang H: miR-92b-3p-TSC1 axis is critical for mTOR signaling-mediated vascular smooth muscle cell proliferation induced by hypoxia. Cell Death Differ 2018.

7. Bandres E, Bitarte N, Arias F, et al: microRNA-451 regulates macrophage migration inhibitory factor production and proliferation of gastrointestinal cancer cells. Clin Cancer Res 15: 2281-2290, 2009.

8. Lynam-Lennon N, Maher SG and Reynolds JV: The roles of microRNA in cancer and apoptosis. Biol Rev Camb Philos Soc 84: 55-71, 2009.

9. Zhang T, Zhang Z, Li F, et al: miR-143 Regulates Memory T Ce11 Differentiation by Reprogramming T Cell Metabolism. J Immunol 201: 2165-2175, 2018.

10. Bi Y, Liu G and Yang R: MicroRNAs: novel regulators during the immune response. J Cel1 Physiol 218: 467-472, 2009.

11. Goradel NH, Mohammadi N, Haghi-Aminjan H, Farhood B, Negahdari B and Sahebkar A: Regulation of tumor angiogenesis by microRNAs: State of the art. J Cell Physiol 234: 1099-1110, 2019.

12. Rangrez AY, Massy ZA, Metzinger-Le Meuth V and Metzinger L: miR-143 and miR-145: molecular keys to switch the phenotype of vascular smooth muscle cells. Circ Cardiovasc Genet 4: 197-205, 2011. 
13. Wang M, Li W, Chang GQ, et al: MicroRNA-21 regulates vascular smooth muscle cell function via targeting tropomyosin 1 in arteriosclerosis obliterans of lower extremities. Arterioscler Thromb Vasc Biol 31: 2044-2053, 2011.

14. Yang F, Chen $\mathrm{Q}$, He $\mathrm{S}$, et al: miR-22 Is a Novel Mediator of Vascular Smooth Muscle Cell Phenotypic Modulation and Neointima Formation. Circulation 137: 1824-1841, 2018.

15. Welch C, Chen $\mathrm{Y}$ and Stallings RL: MicroRNA-34a functions as a potential tumor suppressor by inducing apoptosis in neuroblastoma cells. Oncogene 26: 5017-5022, 2007.

16. Raver-Shapira N, Marciano E, Meiri E, et al: Transcriptional activation of miR-34a contributes to p53-mediated apoptosis. Mol Ce11 26: 731-743, 2007.

17. Chang TC, Wentzel EA, Kent OA, et al: Transactivation of miR-34a by p53 broadly influences gene expression and promotes apoptosis. Mol Cell 26: $745-752,2007$.

18. Chen Q, Yang F, Guo M, et al: miRNA-34a reduces neointima formation through inhibiting smooth muscle cell proliferation and migration. J Mol Cel1 Cardiol 89: 75-86, 2015.

19. Zhang L, He S, Guo S, et al: Down-regulation of miR-34a alleviates mesangial proliferation in vitro and glomerular hypertrophy in early diabetic nephropathy mice by targeting GAS1. J Diabetes Complications 28: 259-264, 2014.

20. Bu P, Chen KY, Chen JH, et al: A microRNA miR-34a-regulated bimodal switch targets Notch in colon cancer stem cells. Cel1 Stem Ce11 12: 602-615, 2013.

21. Jin $\mathrm{M}$, Wu $\mathrm{Y}$, Wang $\mathrm{J}$, et al: MicroRNA-24 promotes 3T3-L1 adipocyte differentiation by directly targeting the MAPK7 signaling. Biochem Biophys Res Commun 474: 76-82, 2016. 
22. Jin $\mathrm{M}$, Wu Y, Wang $\mathrm{Y}$, et al: MicroRNA-29a promotes smooth muscle cell differentiation from stem cells by targeting YY1. Stem Cell Res 17: 277-284, 2016.

23. Zheng $\mathrm{X}$, Wu Y, Zhu L, et al: Angiotensin II promotes differentiation of mouse embryonic stem cells to smooth muscle cells through PI3-kinase signaling pathway and NF-kappaB. Differentiation 85: 41-54, 2013.

24. Li Z, Zhang Q, Wu Y, et al: 1ncRNA Malat1 modulates the maturation process, cytokine secretion and apoptosis in airway epithelial cel1-conditioned dendritic cells. Exp Ther Med 16: 3951-3958, 2018.

25. Wu Y, Li Z, Yang M, et al: MicroRNA-214 regulates smooth muscle cell differentiation from stem cells by targeting RNA-binding protein QKI. Oncotarget 8: 19866-19878, 2017.

26. Zhang L, Chen Q, An W, Yang F, Maguire EM and Chen D: Nove1 Pathological Role of hnRNPA1 (Heterogeneous Nuclear Ribonucleoprotein A1) in Vascular Smooth Muscle Cell Function and Neointima Hyperplasia. 37: 2182-2194, 2017.

27. 0'Brien ER, Garvin MR, Stewart DK, et al: Osteopontin is synthesized by macrophage, smooth muscle, and endothelial cells in primary and restenotic human coronary atherosclerotic plaques. Arterioscler Thromb 14: 1648-1656, 1994.

28. Gadeau AP, Campan M, Millet D, Candresse T and Desgranges C: Osteopontin overexpression is associated with arterial smooth muscle cell proliferation in vitro. Arterioscler Thromb 13: 120-125, 1993.

29. Tardelli M, Zeyda K, Moreno-Viedma V, et al: 0steopontin is a key player for local adipose tissue macrophage proliferation in obesity. Mol Metab 5: 1131-1137, 2016.

30. Bostrom K: 0steopontin, a missing link in PDGF-induced smooth muscle cell migration. Cardiovasc Res 75: 634-635, 2007. 
31. Liaw L, Skinner MP, Raines EW, et al: The adhesive and migratory effects of osteopontin are mediated via distinct cell surface integrins. Role of alpha $\mathrm{v}$ beta 3 in smooth muscle cell migration to osteopontin in vitro. J Clin Invest 95: 713-724, 1995.

32. Alexander MR and Owens GK: Epigenetic control of smooth muscle cell differentiation and phenotypic switching in vascular development and disease. Annu Rev Physiol 74: 13-40, 2012.

33. Cheng W, Yan K, Chen Y, Zhang W, Ji Z and Dang C: ABCA1 inhibits PDGF-induced proliferation and migration of rat airway smooth muscle cell through blocking TLR2/NF-kappaB/NFATc1 signaling. J Cel1 Biochem 119: 7388-7396, 2018.

34. Yasunari K, Kohno M, Kano H, Yokokawa K, Minami M and Yoshikawa $\mathrm{J}$ : Mechanisms of action of troglitazone in the prevention of high glucose-induced migration and proliferation of cultured coronary smooth muscle cells. Circ Res 81: 953-962, 1997.

35. Yasunari K, Kohno M, Kano H, Yokokawa K, Minami M and Yoshikawa $\mathrm{J}$ : Antioxidants improve impaired insulin-mediated glucose uptake and prevent migration and proliferation of cultured rabbit coronary smooth muscle cells induced by high glucose. Circulation 99: 1370-1378, 1999.

36. Aikawa M, Sivam PN, Kuro-o M, et al: Human smooth muscle myosin heavy chain isoforms as molecular markers for vascular development and atherosclerosis. Circ Res 73: 1000-1012, 1993.

37. Schwartz SM, Campbe11 GR and Campbel1 JH: Replication of smooth muscle cells in vascular disease. Circ Res 58: 427-444, 1986.

38. Li H, Telemaque S, Miller RE and Marsh JD: High glucose inhibits apoptosis induced by serum deprivation in vascular smooth muscle cells via upregulation of Bc1-2 and Bc1-x1. Diabetes 54: 540-545, 2005.

39. Hall JL, Chatham JC, Eldar-Finkelman $H$ and Gibbons GH: Upregulation of glucose metabolism during intimal lesion formation is 
coupled to the inhibition of vascular smooth muscle cell apoptosis. Role of GSK3beta. Diabetes 50: 1171-1179, 2001.

40. Chen $\mathrm{F}$ and Hu SJ: Effect of microRNA-34a in cell cycle, differentiation, and apoptosis: a review. J Biochem Mol Toxicol 26: 79-86, 2012.

41. Adams BD, Wali VB, Cheng CJ, et al: miR-34a Silences c-SRC to Attenuate Tumor Growth in Triple-Negative Breast Cancer. Cancer Res 76: 927-939, 2016.

42. Liu YW, Sun M, Xia R, et al: LincHOTAIR epigenetically silences miR34a by binding to PRC2 to promote the epithelial-to-mesenchymal transition in human gastric cancer. Cell Death Dis 6: e1802, 2015.

43. Han Z, Zhang $Y$, Yang Q, et al: $m i R-497$ and miR-34a retard lung cancer growth by co-inhibiting cyclin E1 (CCNE1). Oncotarget 6: 13149-13163, 2015.

44. Chua CEL and Tang BL: miR-34a in Neurophysiology and Neuropathology. J Mol Neurosci 67: 235-246, 2019.

45. He L, He X, Lim LP, et al: A microRNA component of the p53 tumour suppressor network. Nature 447: 1130-1134, 2007.

46. Lei CT, Tang H, Ye C, et al: MDM2 Contributes to High Glucose-Induced Glomerular Mesangial Cell Proliferation and Extracellular Matrix Accumulation via Notch1. Sci Rep 7: 10393, 2017.

47. Tang H, Lei CT, Ye C, et al: MDM2 is implicated in high-glucose-induced podocyte mitotic catastrophe via Notch1 signalling. J Cel1 Mol Med 21: 3435-3444, 2017.

48. Sweeney C, Morrow D, Birney YA, et al: Notch 1 and 3 receptor signaling modulates vascular smooth muscle cell growth, apoptosis, and migration via a CBF-1/RBP-Jk dependent pathway. FASEB J 18: 1421-1423, 2004. 
49. Li Y, Guessous F, Zhang Y, et al: MicroRNA-34a inhibits glioblastoma growth by targeting multiple oncogenes. Cancer Res 69: 7569-7576, 2009.

50. Kang L, Mao J, Tao Y, et al: MicroRNA-34a suppresses the breast cancer stem cel1-like characteristics by downregulating Notch1 pathway. Cancer Sci 106: 700-708, 2015.

51. Tang Y, Tang Y and Cheng YS: miR-34a inhibits pancreatic cancer progression through Snail1-mediated epithelial-mesenchymal transition and the Notch signaling pathway. Sci Rep 7: 38232, 2017.

\section{Figure legends:}

Figure 1. Proliferation and migration of vascular smooth muscle cells (VSMCs), and miR-34a expression under high glucose stimulation. (A and B) VSMCs cultured in HG medium showed a greater growth density compared with NG group under microscopy. (C and D) Transwell migration analyses of VSMCs cultured in HG or NG medium for 24 hours. (E) miR-34a expression of VSMCs cultured in HG medium or NG medium (0 hour) determined at different timepoints using RT-qPCR. Data is representative or means \pm S.E.M. of at least three independent samples. $* \mathrm{P}<0.05, * *$ $\mathrm{P}<0.01$.

Figure 2. High glucose-induced vascular smooth muscle cell(VSMC) proliferation and migration are impaired by miR-34a mimic. (A-B) miR-34a mimic reduced HG induced VSMC migration while the migrated cells between $\mathrm{NG}+$ mimic $\mathrm{NC}$ group and $\mathrm{NG}+$ miR-34a mimic group seemed no significance. (C) VSMC transfected with mimic NC or miR-34a mimic respectively were cultured in NG or HG medium for 24 hours and then subjected to CCK8 analyses. (D and E) VSMC transfected with mimic NC or miR-34a mimic respectively were cultured in NG or HG medium for 12 hours. Then, cells were harvested to examine the OPN and PCAN 
mRNA expression levels by RT-qPCR. Data is representative or means \pm S.E.M. of at least three independent samples. ${ }^{*} \mathrm{P}<0.05,{ }^{*} * \mathrm{P}<0.01$.

Figure 3. High glucose-induced downregulation of VSMC specific markers was modulated by miR-34a mimic or miR-34a Int mimic. (A-D) Expression of VSMC specific markers SM22, SMA and SMMHC were detected. Data is representative or means \pm S.E.M. of at least three independent samples. $* \mathrm{P}<0.05, * * \mathrm{P}<0.01$.

Figure 4. miR-34a regulated high glucose-induced VSMC proliferation and migration through its target gene Notch1. (A) The potential wild type binding sites of miR-34a within Notch1 3'-UTR predicted by TargetScan. (B - D) VSMCs were transfected with miR-34a mimic or miR-34a Int mimic and their NC for 48 hours and then harvested for western blot and RT-qPCR. (E and F) Transfection of siRNANotch1 into VSMCs significantly downregulated Notch1 mRNA and protein level. (G) miR-34a Int mimic and siRNA- Notch1 were co-transfected into VSMCs cultured in HG. Cell counting assays showed miR-34a Int induced VSMC proliferation was eliminated by inhibition of Notch1 in these cells. Data is representative or means \pm S.E.M. of at least three independent samples. $* \mathrm{P}<0.05, * * \mathrm{P}<0.01$. 


\section{Figures}

A

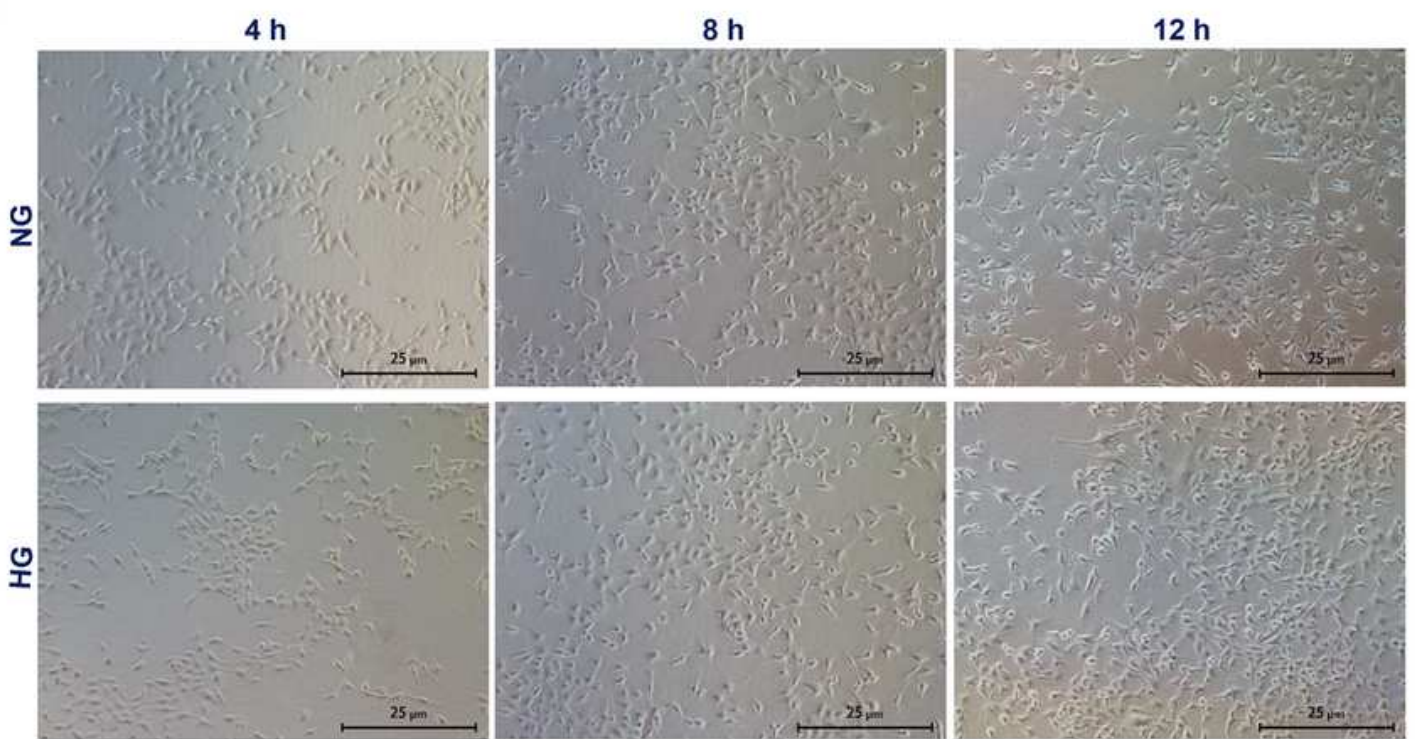

B

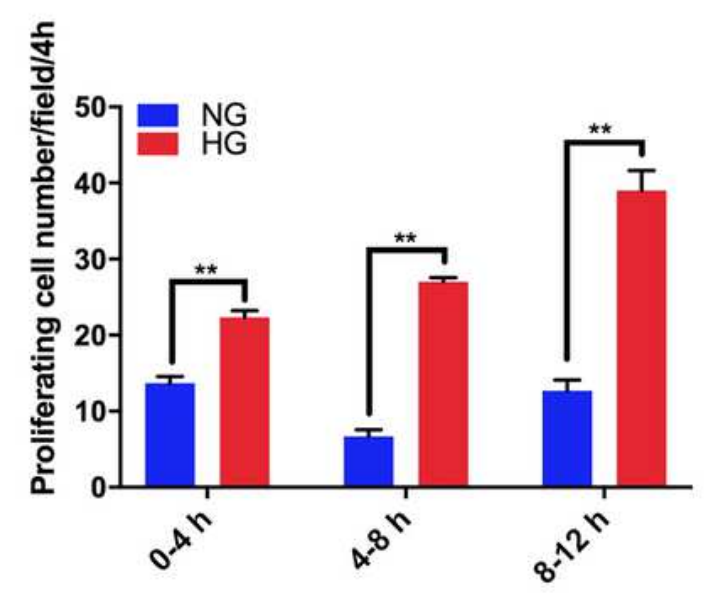

D

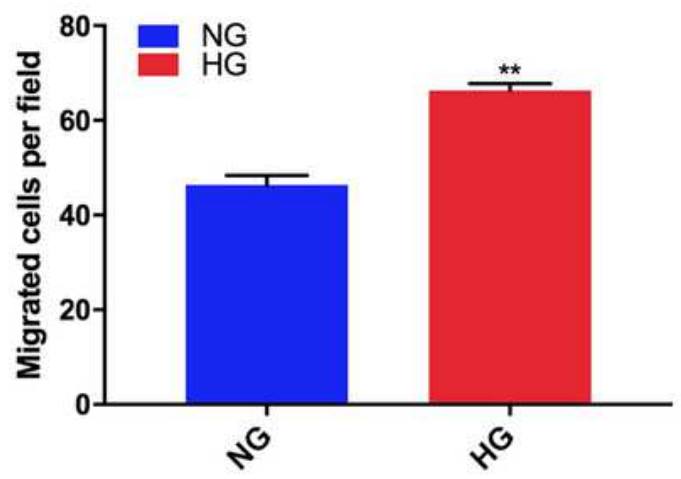

C

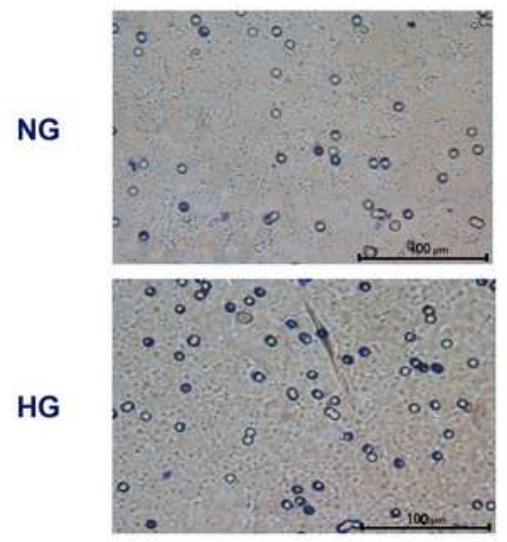

E

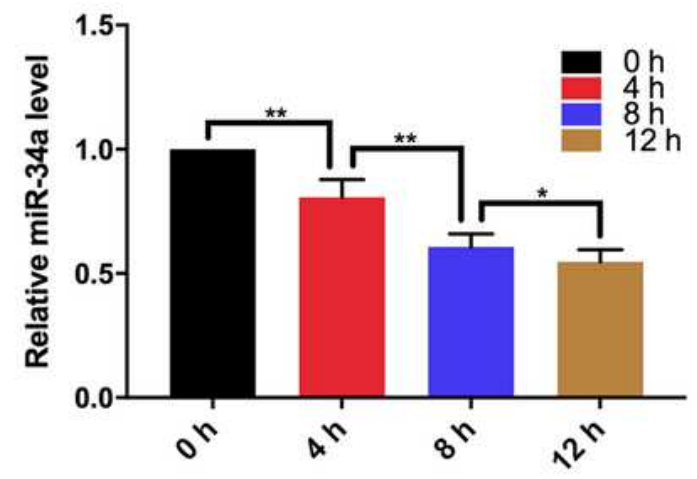

Figure 1

Proliferation and migration of vascular smooth muscle cells (VSMCs), and miR-34a expression under high glucose stimulation. (A and B) VSMCs cultured in HG medium showed a greater growth density compared with NG group under microscopy. (C and D) Transwell migration analyses of VSMCs cultured 
in HG or NG medium for 24 hours. (E) miR-34a expression of VSMCs cultured in HG medium or NG medium (0 hour) determined at different timepoints using RT-qPCR. Data is representative or means \pm S.E.M. of at least three independent samples. * $P<0.05, * \star P<0.01$.

A
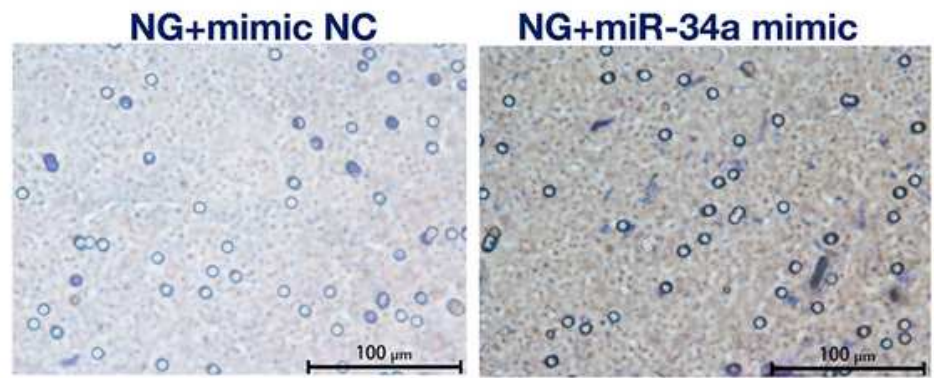

$\mathrm{HG}+$ mimic NC

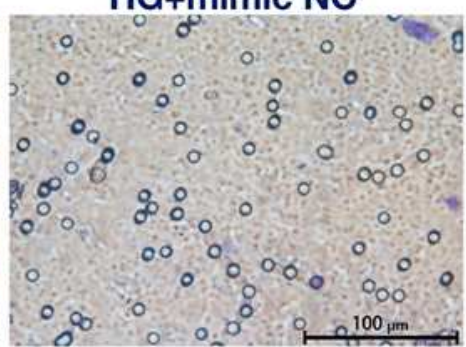

HG+miR-34a mimic

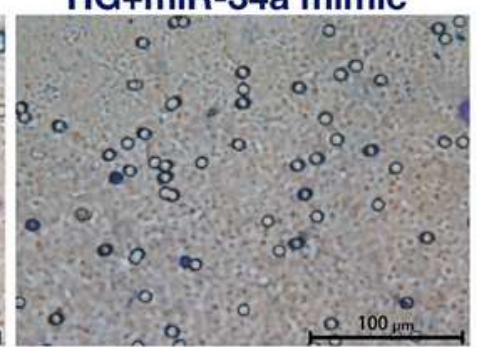

B

C
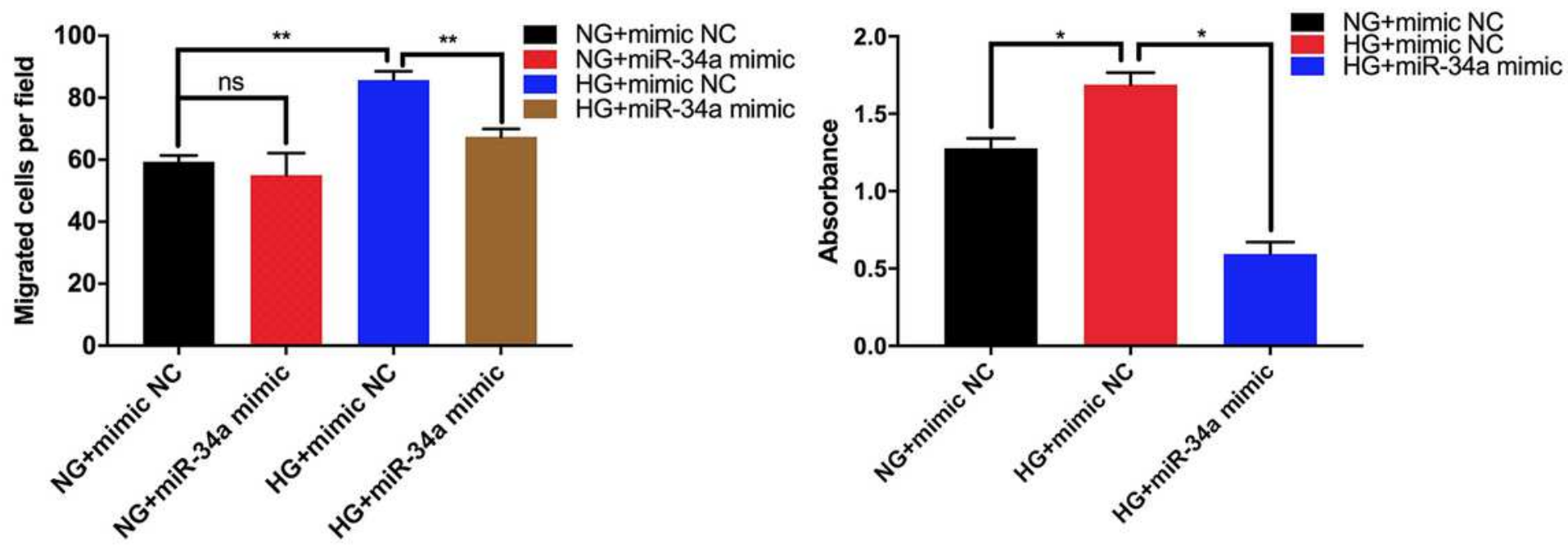

D

$\mathbf{E}$
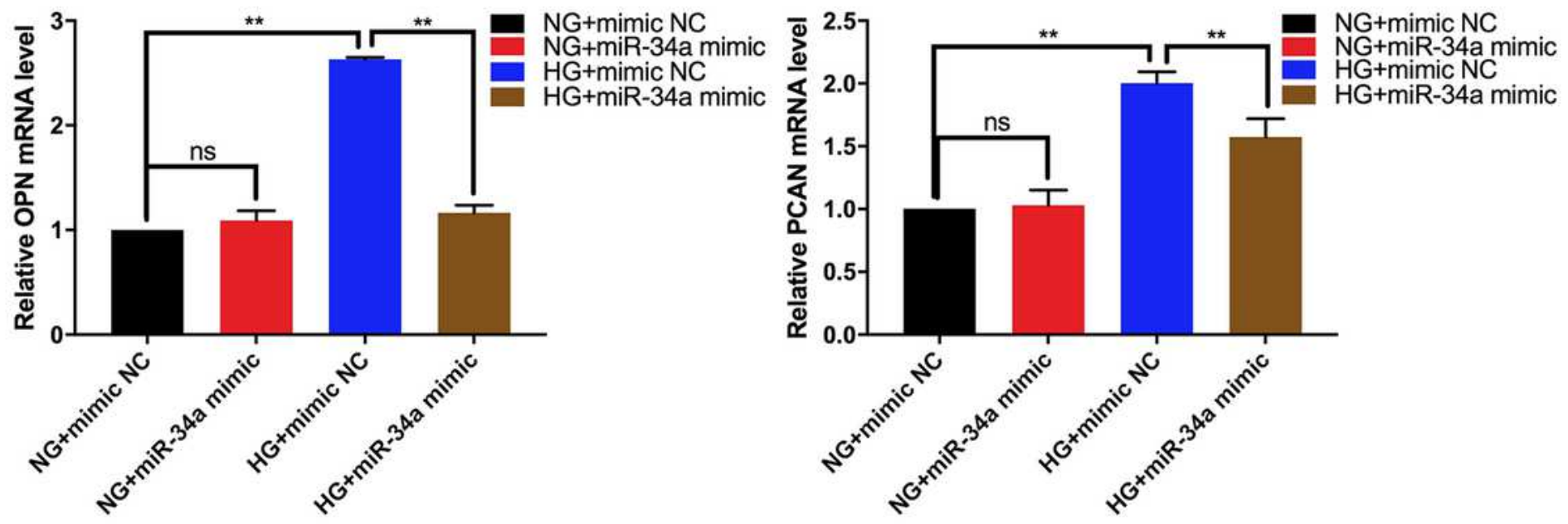

Figure 2 
High glucose-induced vascular smooth muscle cell(VSMC) proliferation and migration are impaired by miR-34a mimic. (A-B) miR-34a mimic reduced HG induced VSMC migration while the migrated cells between NG+mimic NC group and NG+miR-34a mimic group seemed no significance. (C) VSMC transfected with mimic NC or miR-34a mimic respectively were cultured in NG or HG medium for 24 hours and then subjected to CCK8 analyses. (D and E) VSMC transfected with mimic NC or miR-34a mimic respectively were cultured in NG or HG medium for 12 hours. Then, cells were harvested to examine the OPN and PCAN mRNA expression levels by RT-qPCR. Data is representative or means \pm S.E.M. of at least three independent samples. ${ }^{*} P<0.05,{ }^{\star} \mathrm{P}<0.01$.

A
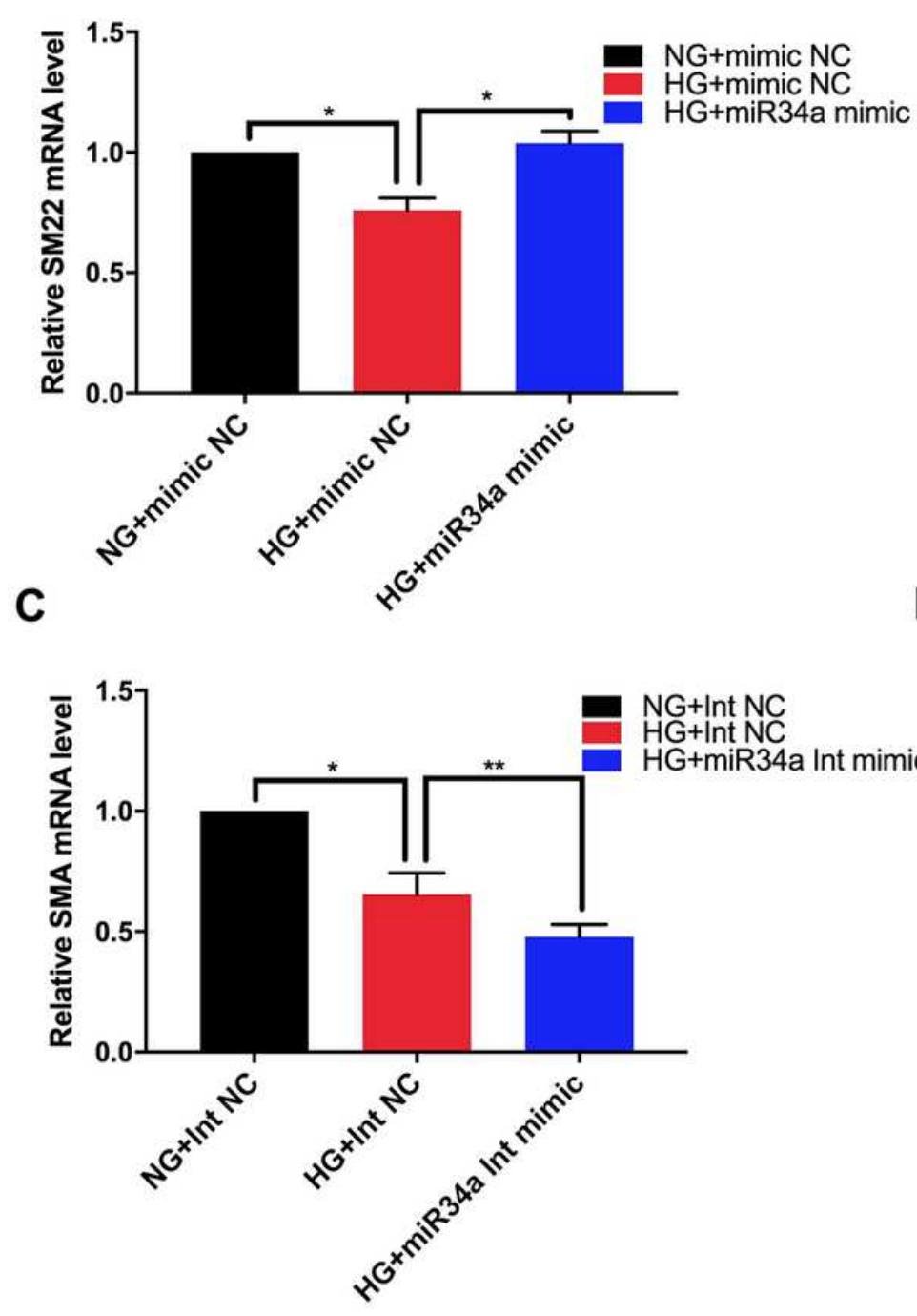

B
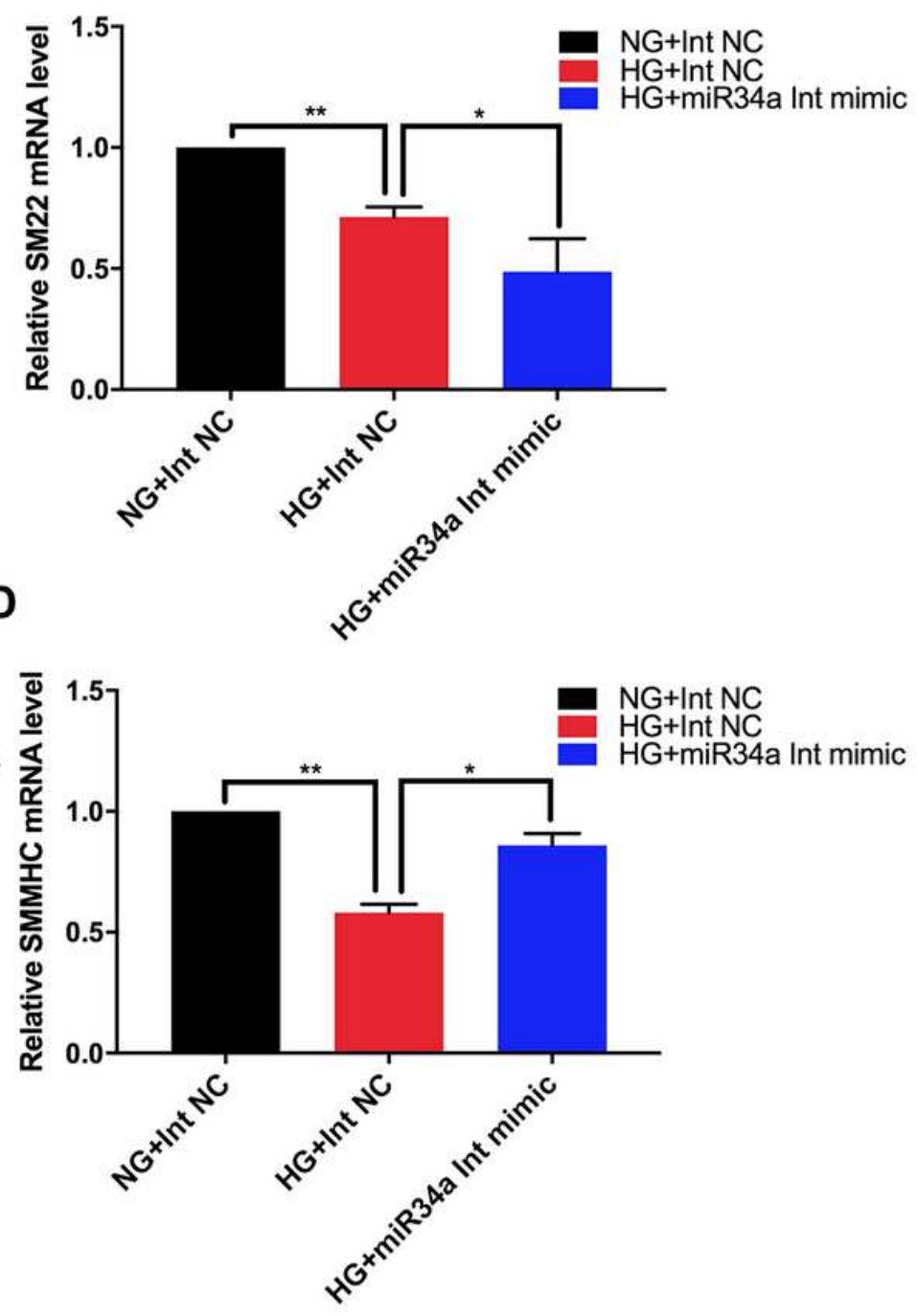

Figure 3

High glucose-induced downregulation of VSMC specific markers was modulated by miR-34a mimic or miR-34a Int mimic. (A-D) Expression of VSMC specific markers SM22, SMA and SMMHC were detected. Data is representative or means \pm S.E.M. of at least three independent samples. ${ }^{*} P<0.05,{ }^{\star} * P<0.01$. 
A

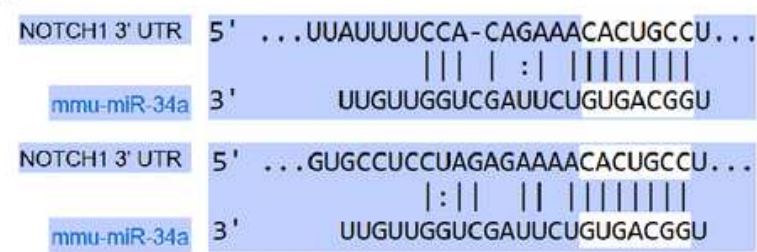

C

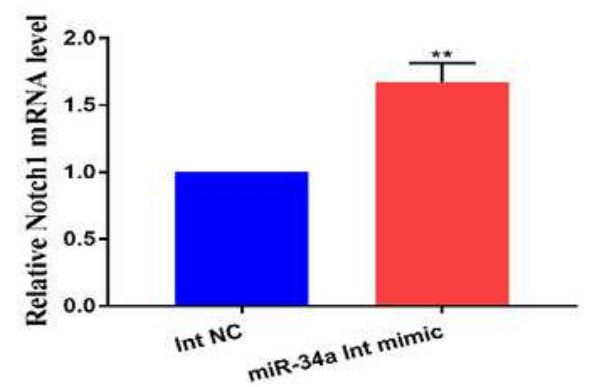

E

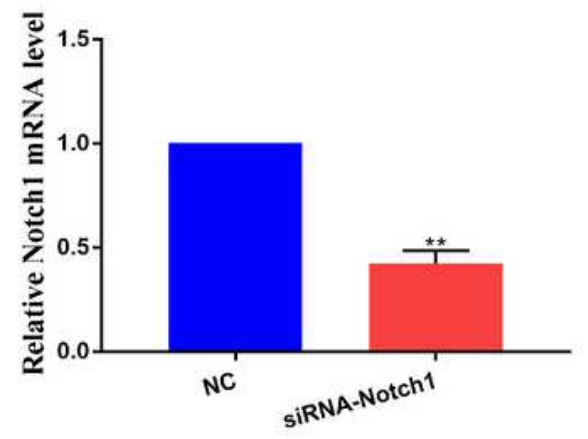

G

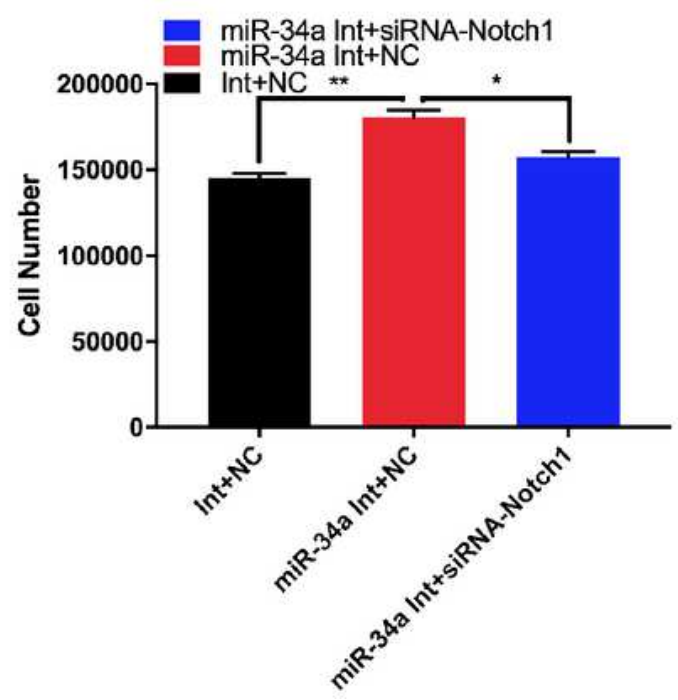

B

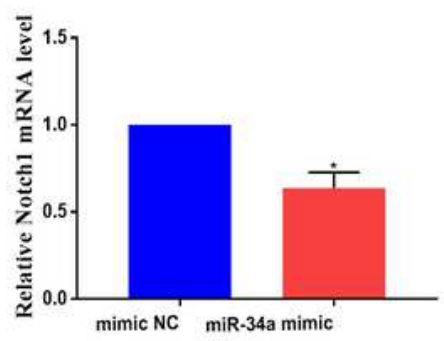

D

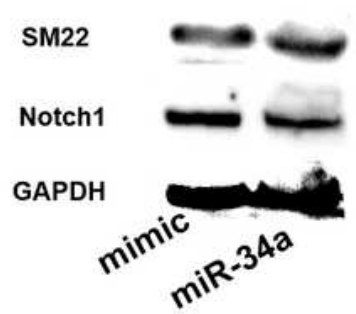

F

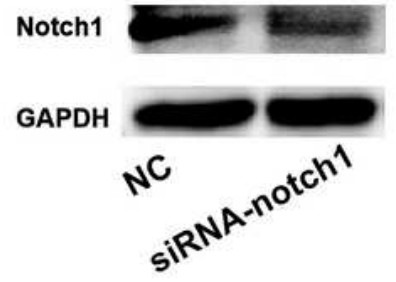

\section{Figure 4}

miR-34a regulated high glucose-induced VSMC proliferation and migration through its target gene Notch1. (A) The potential wild type binding sites of miR-34a within Notch1 3 ' -UTR predicted by TargetScan. (B - D) VSMCs were transfected with miR-34a mimic or miR-34a Int mimic and their NC for 48 hours and then harvested for western blot and RT-qPCR. (E and F) Transfection of siRNANotch1 into VSMCs significantly downregulated Notch1 mRNA and protein level. (G) miR-34a Int mimic and siRNA- 
Notch1 were co-transfected into VSMCs cultured in HG. Cell counting assays showed miR-34a Int induced VSMC proliferation was eliminated by inhibition of Notch1 in these cells. Data is representative or means \pm S.E.M. of at least three independent samples. ${ }^{\star} P<0.05,{ }^{* *} P<0.01$. 